\title{
Una estrategia didáctica que promueve el desarrollo de la competencia matemática comunicar en el contexto cafetero ${ }^{1}$
}

\author{
A teaching strategy that promotes the development of \\ mathematical competence coffee communicate in the context
}

\section{Uma estratégia de ensino que promove o desenvolvimento de cálculo competência comunicam café no contexto}

Recibido: mayo de 2013

Aceptado: agosto de 2013
Shirly Zúñiga Arredondo ${ }^{2}$

Víctor Bolaños Ortizo ${ }^{3}$

Dawson Didier Cortés Joven ${ }^{4}$

\section{Resumen}

En una investigación que se adelanta con estudiantes de noveno grado de la I. E. Guacacallo del Municipio de Pitalito, se pretende aplicar una estrategia didáctica que apoyada en un modelo teórico a priori, articula el aprendizaje del conocimiento de la función cuadrática en aspectos afectivos y cognitivos para permitir el desarrollo de la competencia matemática comunicar. Teniendo en cuenta la postura sociocultural de competencia, como una propuesta educativa que trasciende el carácter funcional de la matemática y que propende por una formación integral de los estudiantes desde la interacción en el aula, y el uso social de la competencia en aprendizajes situados, dando solución a problemas contextualizados, como en este caso, referente a la producción y comercialización del café.

Palabras clave: Estrategia didáctica; Competencia Matemática Comunicar; Modelo teórico a priori; matemáticas escolares; álgebra; funciones polinómicas; función Cuadrática; investigación e innovación en educación matemática; teorías de aprendizaje; situado sociocultural.

\begin{abstract}
In an investigation conducted with students from ninth grade I. E. Pitalito Guacacallo Township, is intended to apply to a teaching strategy supported by a theoretical model a priori knowledge articulated learning quadratic
\end{abstract}

1 Artíulo de Investigación

2 Universidad de la Amazonia, Maestría en Ciencias de la Educación con Énfasis en Didáctica de la Matemática Contacto: shirlyzuniga@gmail.com

3 Universidad de la Amazonia, Maestría en Ciencias de la Educación con Énfasis en Didáctica de la Matemática Contacto: victor235711@hotmail.com

4 Universidad de la Amazonia, Maestría en Ciencias de la Educación con Énfasis en Didáctica de la Matemática Contacto: dawsondidier@gmail.com 
function in affective and cognitive aspects to enable the development of mathematical competence to communicate. Considering the competition sociocultural position as an educational proposal that transcends the functional character of mathematics and aims for comprehensive training of students from the classroom interaction and the social use of situated learning competition, giving solution to problem situations, as in this case, concerning the production and marketing of coffee.

Keywords: Teaching strategy; Communicating Mathematics Competition; priori theoretical model; school math, algebra, polynomial functions, Quadratic function, research and innovation in mathematics education, learning theories, situated sociocultural.

\section{Resumo}

Em uma investigação realizada com alunos da nona série I. E. Pitalito Guacacallo Township, deverá aplicar-se a uma estratégia de ensino suportado por um modelo teórico de um conhecimento a priori articulada aprendendo função quadrática em aspectos afetivos e cognitivos para permitir o desenvolvimento de competência matemática para se comunicar. Considerando a posição sociocultural competição como uma proposta educativa que transcende o caráter funcional da matemática e objetivos para a formação integral dos alunos a partir da interação em sala de aula eo uso social da competição aprendizagem situada, dando solução de situações-problema, como neste caso, relativo à produção e comercialização de café.

Palavras-chave: estratégia de ensino; Comunicação da Matemática Concorrência; priori modelo teórico; matemática do ensino médio, álgebra, funções polinomiais, função quadrática, investigação e inovação em educação matemática, as teorias de aprendizagem, situado sociocultural.

\section{Planteamiento del problema}

Dado el gran interés en los últimos años del estudio en competencias a nivel internacional y en consecuencia a nivel nacional, se ha dado un giro educacional en Colombia pasando de un currículo centrado en contenidos a una visión basada en el enfoque por competencias, plasmado en un contexto social, dotado de situaciones donde la interacción entre los participantes de la clase son el eje principal para promover el desarrollo de competencias. Es necesario y urgente que los docentes diseñen estrategias de aprendizaje que contribuyan a postular un conocimiento construido y compartido social y culturalmente; además, de mostrar la utilidad de la competencia en un contexto social, para que el profesor comprenda mejor las matemáticas y la asuma para promover desde sus prácticas de enseñanza una pragmática de uso, aprendizajes situados y solución de problemas contextualizados, como el que se tiene en cuenta para este caso, referenciar para su desarrollo en el aspecto cognitivo, el trabajo con la producción y comercialización del café, en el estudio de la función cuadrática.

Incorporar a la estrategia de aprendizaje un modelo teórico a priori (MTAP), en el que se involucran tareas, procesos matemáticos y niveles de complejidad, Solar (2009), adheridos a unos procesos de comunicación, será clave para promover el desarrollo de la competencia matemática comunicar (CMC), y así, contribuir a fortalecer la inclusión de las competencias al currículo de matemáticas, y 
ayudar a formalizar el carácter de instrucción y no de formación en el estudiante, de manera integral, dejando de lado el sujeto pasivo valorado solamente desde aspectos cognitivos, y en el cual tiene que ver en un alto porcentaje con la problemática a la hora de incorporar competencias a los nuevos procesos de aprendizaje. Por ello, nos hemos propuesto dar respuesta a la pregunta ¿Cómo formular e implementar una propuesta didáctica que contribuya a desarrollar la competencia matemática comunicar asociada al aprendizaje del objeto matemático función cuadrática, en estudiantes de grado noveno de la I. E. Guacacallo del Municipio de Pitalito (Huila)?

\section{Referentes conceptuales}

Los referentes teóricos en los que se basa la investigación se centra en estudios que hacen diversos autores acerca de incorporar al currículo de matemáticas un enfoque basado en el desarrollo de competencias, mediante la aplicación de MTAP, que permita promover el desarrollo de la CMC, relacionado al estudio del objeto matemático función cuadrática, en su significado, fenomenología, sistemas de representación en el contexto cafetero del municipio de Pitalito; inicialmente asumimos la conceptualización de CMC a partir de diversos aspectos que aparecen en el estudio de la interacción en el aula de matemáticas, donde se involucren procesos comunicacionales y elementos que componen de manera general la competencia; tales como, los planteados por Bishop (2005), Rico y Lupiañez (2008), Sfard (2008), D'amore, Godino y Fandiño (2005), y Arévalo (2012). Los anteriores estudios sirvieron para tomar postura en la investigación de la CMC de la siguiente manera:

La CMC ayuda al estudiante en el desarrollo y el proceso de la negociación en un discurso matemático que genere aspectos primordiales de la comunicación escrita y verbal, convirtiéndose en un miembro activo y participante de la comunidad de aprendizaje, compartiendo y desarrollando el significado matemático en situaciones contextuales, $y$ utilizarlo socialmente desde la relación entre factores meta-cognitivos, afectivos y volitivos.

En cuanto al MTAP, se involucran los aspectos mencionados como componentes de la competencia matemática, referidos por D'amore et al., (2005), cognitivo (relacionado con el estudio del objeto matemático, Función cuadrática), el afectivo (involucrado al proceso matemático de la motivación) y de tendencia a la acción (analizado con el proceso matemático de la dedicación), combinado con los expuestos por Solar (2009): Procesos matemáticos, Tareas y Niveles de Complejidad. Para el caso del aspecto cognitivo se involucran los procesos matemáticos, de Participación, Negociación y Actividad discursiva, como procesos propios de la interacción social en el aula de matemáticas como entes de la actividad comunicacional que permite construir significados matemáticos, comunicarlos, compartirlos y desarrollarlos como estudiantesparticipantes miembros activos de una comunidad de aprendizaje (Bishop, 2005 y Sfard, 2008).

En el caso del objeto matemático Función Cuadrática y considerando a Villa (2008) quien plantea el estudio de la función en el desarrollo del pensamiento variacional a través de la educación básica y secundaria, donde tiene relación con la producción de café como lo demuestran diversas investigaciones a nivel nacional; se cita en Perdomo y Heuth (2011), quienes asumen el modelo cuadrático en diversos cultivos en lo que comúnmente se denomina cosecha, delineando dicha función en su época de mayor producción. El objeto matemático se define en Villa (2008) "Se llama función cuadrática a la relación entre dos cantidades de magnitud cuya razón de cambio varía linealmente" (p. 248).

\section{Metodología}

La Investigación es de carácter cualitativa, orientada en el Enfoque Sociocultural de naturaleza aplicada, los resultados serán analizados con base 
a la propuesta didáctica del desarrollo de CMC. El método de Investigación a seguir es el análisis del estudio de caso. Para el cual, se tendrá en cuenta los episodios de clase más significativos, correspondiente al trabajo realizado por cuatro estudiantes, que serán tenidos en cuenta para valorar las capacidades, descriptores y actuaciones, durante la actividad matemática y observar la aplicabilidad de la estrategia didáctica en la movilización de la CMC. El apoyo de algunas técnicas de investigación, servirán para afianzar la recolección y posterior análisis de la información, obtenida de videos, audios, pruebas pilotos, entrevistas, cuestionarios y análisis de documentos y materiales que serán utilizados en el muestreo para su respectiva codificación.

\section{Análisis de datos}

Recogida la información, se analizará episodios de clase, en los cuales ocurra con mayor frecuencia aspectos del proceso comunicativo, correspondiente a la actividad matemática desarrollada por los cuatro estudiantes sujetos de estudio. El análisis de los episodios se basará en el resumen de los descriptores y las actuaciones sucedidos en cada situación matemática presentada en la tarea y que hacen parte de los componentes del MTAP, cada situación matemática presenta para su desarrollo un nivel creciente de complejidad de la CMC. Después de manera general se realizará mediante rejillas de valoración para cada componente del MTAP, y en especial, de los procesos matemáticos, el respectivo proceso de evaluación, y describir al final la efectividad de la estrategia de aprendizaje. A continuación se muestra una actividad que se desarrollará en la investigación.

Entre las distintas especies de café el caturra fue uno de los mayores exponentes en la década pasada, por sobresalir en mayor cantidad de producción, en la anterior gráfica se muestra la producción en arrobas de café pergamino seco por hectárea (Producción @ c.p.s. / ha) sobre la edad en cultivo en años, datos correspondientes a un cultivo de 5.000 palos por hectárea, según la información anterior responda las siguientes preguntas:
Figura 1

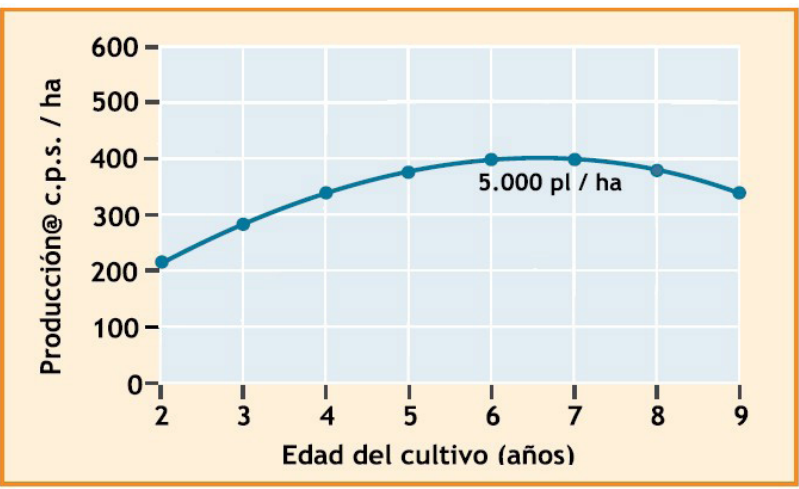

Fuente: Tomada de Perdomo y Hueth (2011) donde indica la productividad de la variedad caturra según la edad y la densidad de 5.000 palos por hectárea, en cafetales a plena exposición solar y a libre crecimiento Tomada de Perdomo y Hueth (2011) donde indica la productividad de la variedad caturra según la edad y la densidad de 5.000 palos por hectárea, en cafetales a plena exposición solar y a libre crecimiento

1. Comente con sus compañeros sobre otra forma de representar la información de la gráfica.

2. Qué relación se puede establecer entre los datos presentados.

3. Qué relación hay presente entre la edad del cultivo y lo producción del cultivo, expréselo de manera escrita y luego compártalo con su profesor.

4. Entre los 5 y 6 años de cultivado el café que sucede con su producción.

5. Exponga y argumente qué sucederá con un cafetal pasado nueve años de haberse cultivado.

Posterior a la actividad matemática, y a partir de unos episodios de clase significativos en cuanto a los procesos de comunicación, se sistematizara por códigos los descriptores correspondiente a cada procesos matemático, y analizar el nivel de complejidad (PISA ,2006: Reproducción, conexión y reflexión) alcanzado por la CMC, y por último valorar la aplicabilidad de la estrategia de aprendizaje puesta en marcha. 
ral a la educación matemática. Santiago de

\section{Conclusiones}

Los resultados de la investigación permitirá a futuro y a corto plazo incorporar al currículo de matemáticas estrategia didácticas que permitan en el aprendizaje desarrollar la competencia matemática comunicar, mediante la utilización de un modelo teórico a priori, en el que se relaciona las tareas, procesos matemáticos y niveles de complejidad, con factores de carácter metacognitivo, volitivo y de tendencia a la acción, aspectos primordiales dentro de un contexto social para comprender las matemáticas. Además, se espera que surjan diversas investigaciones que deben emerger de esta propuesta de investigación en el futuro desarrollo de competencias matemáticas en un contexto situado.

\section{Referencias}

Arcila, J. (2007). Renovación y administración de los cafetales para estabilizar la producción de la finca. En J. Arcila, F. Farfán, A. Moreno, L. Salazar, \& E. Hincapié, Sistemas de producción de café en Colombia (págs. 144-160). Chinchina, Caldas.

Arévalo, A. (2012). Comunicación matemática en el aula: uso y gestión de estrategias. Tesis de maestría, Universidad Católica de la santísima Concepción, Concepción.

Bishop, A. (2005). Aproximación sociocultu-
D Amore, B., Godino, J., \& Fandiño, M. (2005). Competencias y matemáticas. Bogotá: Editorial magisterio.

Perdomo, J., \& Hueth, D. (2011). Funciones de producción, análisis de economías a escala y eficiencia técnica en el eje cafetero colombiano: una aproximación con frontera estocástica. Revista colombiana de estadística, 34 (2), 377-402.

Rico, L., \& Lupiañez, J. (2008). Competencias matemáticas desde una perspectiva curricular. Madrid: Alianza editorial.

Sfard, A. (2008). Aprendizaje de las matemáticas escolares desde un enfoque comunicacional. Cali: Universidad del Valle.

Solar, H. (2009). Competencias de modelización y argumentación en interpretación de gráficas funcionales: propuesta de un modelo de competencias aplicando a un estudio de caso. Tesis de doctorado, Universidad Autónoma de Barcelona, Bellaterra.

Villa, J. (2008). El concepto de función: una mirada desde las matemáticas escolares. Comité Latinoamericano De Matemática Educativa Clame, Congreso llevado a cabo en la Reunión Latinoamericana de Matemática Educativa 21, (págs. 245-254). Estado de México. 